\title{
Ingenio, Uso Hipotético de la Razón y Juicio Reflexionante en la Filosofía de Kant ${ }^{1}$
}

\section{Wit, Hypothetical Use of Reason and Reflecting Power of Judgment in Kant's Philosophy}

\author{
Manuel SÁNCHEZ RodRíGUEZ \\ Universidad de Granada
}

Recibido: 31-12-2011

Aceptado: 23-03-2012

\section{Resumen}

En este artículo se investiga el trasfondo histórico y sistemático de la teoría del Juicio reflexionante de Kant a partir de un estudio histórico del concepto de ingenio [ingenium, Witz]. Aunque los Apuntes de Lecciones de Antropología exponen aún el significado de este concepto en el contexto de la psicología empírica de Baumgarten, estos materiales nos ayudan a interpretar el concepto de ingenio como uno de los precedentes más importantes de la teoría del Juicio reflexionante planteada por Kant en la Crítica del Juicio. La exposición sobre el ingenio pone de manifiesto además la vinculación sistemática e histórica de la tercera Crítica con la teoría del uso hipotético de la razón que encontramos en el Apéndice a la Dialéctica Trascendental de la Crítica de la razón pura.

Palabras clave: Baumgarten, gusto, ingenio, Juicio reflexionante, Kant, Witz.

\begin{abstract}
This article researches the historical and systematic background of Kant's reflecting power of judgment theory based on a historical study of the concept of wit [ingenium, Witz]. Although the Notes from Lessons on Anthropology even

${ }^{1}$ Con la financiación del proyecto de investigación "Leibniz en español" (Ministerio de Ciencia e Innovación, FFI2010-15914, y Junta de Andalucía, P09-HUM-5109). Agradezco a los dos evaluadores de la revista las observaciones y sugerencias enviadas, que han permitido corregir y mejorar la primera versión propuesta.
\end{abstract}


expound the meaning of this concept in the context of Baumgarten's empirical psychology, this material helps us interpret the concept of wit as one of the most important precedents of the reflecting power of judgment theory presented in Kant's Critique of the Power of Judgment. The exposition of wit furthermore highlights the systematic and historic connection between the third Critique and the theory of the hypothetical use of reason found in the Appendix of the Transcendental Dialectic of the Critique of Pure Reason.

Keywords: Baumgarten, Kant, reflecting power of judgment, taste, wit, Witz.

\section{Trasfondo histórico del concepto de ingenio en los Apuntes de Antropología de Kant}

Un estudio sobre el significado del concepto de ingenio [ingenium, Witz] en la filosofía de Kant se enfrenta en primer lugar con el problema de si tal concepto llega a jugar un papel en la teoría criticista de las facultades. En la Crítica de la razón pura las facultades de conocer dejan de ser tratadas meramente como capacidades psicológicas o fuerzas de la razón que puedan ser analizadas respectivamente desde la psicología empírica o la psicología racional. Más bien, su análisis interesa a Kant en tanto que éstas ofrecen o representan en sí mismas condiciones de posibilidad de nuestro conocimiento en general. Aunque la sensibilidad, la imaginación, el ingenio, el entendimiento o el Juicio [Urteilskraft] son, ciertamente, capacidades psicológicas del ánimo, sometidas en cada caso a determinadas reglas empíricas o psicológicas, la crítica exige en particular aislar las condiciones de las mismas que son independientes de toda experiencia y posibilitan a priori nuestro conocimiento de los objetos de la experiencia. Ahora bien, un análisis trascendental del ingenio no tiene lugar en la primera Crítica.

Este concepto, además, aparece con poca frecuencia en la obra publicada de Kant, a diferencia de los Apuntes de Lecciones de Antropología, que incluso dedican al ingenio un capítulo específico junto con el Juicio o la capacidad de juzgarn (Urteilskraft). Kant seguía la sección dedicada a la psicología empírica expuesta en la Metafisica de Baumgarten como guía para su actividad docente en sus Lecciones de Antropología, ${ }^{2}$ de tal modo que tales exposiciones, recogidas en los Apuntes de sus estudiantes, se veían influenciadas por la filosofía baumgartiana en lo que respecta a la estructura y el contenido. Por este motivo, el análisis comparado con la

\footnotetext{
${ }^{2}$ Cf. V-Menschenkunde, AA 25, 889. Se citarán las obras de Kant según la edición de la AkademieAusgabe y el formato usado por la Kant-Gesellschaft (Immanuel Kant. Kant's gesammelte Schriften. Ed. de la Königlich Preußischen Akademie der Wissenschaften und ihren Nachfolgern. Berlin y Leipzig, 1900ss.).
} 
Metafisica de Baumgarten es una condición previa indispensable de la investigación histórica de los Apuntes de Lecciones de Antropología, en la medida en que este manual fue usado por Kant durante aproximadamente cuatro décadas. ${ }^{3}$ Sin embargo, a partir de este análisis puede comprobarse que esta dependencia con respecto al manual no determina completamente el significado específico del concepto de ingenio en tales materiales. Pues ya en su lección del semestre de invierno de 1772/1773 Kant presentaba sus reflexiones sobre este concepto en explícita confrontación con la teoría de Baumgarten: ${ }^{4}$

Nuestro autor [es decir, Baumgarten] ha opuesto el ingenio a la agudeza [Scharfsinnigkeit], y explica el primero mediante una facultad de lo semejante, mientras que la segunda mediante una facultad de conocer la diferencia de las cosas. Pero es mejor oponer el Juicio al ingenio [...]. Al Juicio también le pertenece la facultad de inteligir la coincidencia de las relaciones [...]. El ingenio es la facultad de comparar; el Juicio, la facultad de conectar o de separar. ${ }^{5}$

A diferencia del ingenio y del Juicio, la agudeza no es para Kant una facultad específica del ánimo, sino un grado determinado de refinamiento o perfección que eventualmente puede llegar a alcanzar el sujeto tanto en el uso de su ingenio como de su Juicio. ${ }^{6}$ Por otro lado, mientras que Baumgarten caracteriza la agudeza sólo como una facultad de discernimiento de lo diferente, en la antropología de Kant el Juicio puede inteligir también la conformidad o conexión entre las relaciones o

\footnotetext{
${ }^{3}$ Sin embargo, los editores del volumen 25 de la Akademie-Ausgabe apenas tienen en consideración precisamente esta fuente en su edición crítica de tales materiales, tal como ha destacado Hinske en su recensión de tal edición; Norbert Hinske. "Kant und Alexander Gottlieb Baumgarten. Ein leider unerledigtes Thema der Anthropologie Kants". Aufklärung, 14, 2002, 261-74: especialmente 271.

${ }^{4}$ Baumgarten define el ingenio [ingenium] como la facultad de percibir semejanzas entre las cosas; cf. Alexander Gottlieb BaUmgarten. Metaphysica. Halle, 1739 (reimpresión según la cuarta edición de 1757, en AA 15, 5-54 y AA 17, 5-226): $\S$ 572-75, AA 15, 22s. Al ingenio éste opone la agudeza [acumen] en lugar del Juicio, cf. § 573, AA 15, 22s.

${ }_{5} V$-Anth/Parow, AA 25, 341. La oposición entre ingenio y Juicio la encontramos ya en John Locke. An Essay Concerning Human Understanding. Works of John Locke, 10 vols. London, 1823 ('1690) (reimpresión en Aalen, 1963): vol. 1, libro 2º cap. 11, § 2: 144s., así como en el ensayo Analyse des Genie (1757), cuya traducción al alemán se publicó en 1773 (Johann Georg Sulzer. Entwicklung des Gebriffs von Genie. Vermischte philosophische Schriften. Hildesheim, 1974 ( $\left.{ }^{1} 1773\right)$, 307-22). Una reconstrucción histórico-evolutiva del concepto de ingenio en la ilustración alemana la encontramos en el estudio clásico de Alfred BAeumLer. Das Irrationalitätsproblem in der Ästhetik und Logik des 18. Jahrhunderts bis zur Kritik der Urteilskraft. Vol. 1: Kants Kritik der Urteilskraft. Ihre Geschichte und Systematik. Tübingen, 1923: 141-87, quien, no obstante, no recoge la obra de Sulzer. A pesar de que el alcance y las funciones del ingenio, el Juicio y la sagacidad se describen de forma diferente en Baumgarten y Kant, Vladimir SATURA. Kants Erkenntnispsychologie in den Nachschriften seiner Vorlesungen über empirische Psychologie. Bonn, 1971: 149-51, señala la concepción baumgartiana del Juicio como la fuente principal de la teoría kantiana del Juicio.

${ }^{6}$ Cf. V-Anth/Collins, AA 25, 132s.; V-Anth/Parow, AA 25, 341; V-Anth/Fried, AA 25, 516; V-Anth/ Busolt, AA 25, 1459.
} 
comparaciones que ofrece previamente el ingenio, pues "las cosas semejantes no se encuentran aún conectadas"7 según un fundamento.

\section{El ingenio en la Crítica de la razón pura}

Con todo, en principio debe relativizarse el significado de esta corrección frente a Baumgarten al constatar que ésta no es incorporada con claridad a la Crítica de la razón pura, en la que Kant llega incluso a exponer el concepto de ingenio conservando precisamente alguno de los aspectos criticados previamente a Baumgarten. En su explicación sobre el principio de los géneros y las especies que tiene lugar en el Apéndice a la Dialéctica Trascendental, Kant opone al ingenio la agudeza o la facultad de discernimiento [Unterscheidungsvermögen], en lugar del Juicio, con lo cual parece no incorporar la crítica a Baumgarten expuesta en los Apuntes. Según Kant, la agudeza puede conocer "la multiplicidad y diferencia de las cosas, con independencia de su conformidad bajo el mismo género". Mientras que la "ligereza" del ingenio "permite pensar" las semejanzas de las cosas bajo un género mediante la comparación, ${ }^{9}$ la agudeza determina el contenido en la misma especie "con la intención puesta en la multiplicidad de las especies"10. De este modo, el trabajo conjunto de ingenio y agudeza posibilita el establecimiento de un conocimiento empírico determinado según géneros y especies en el sistema de la experiencia.

Ahora bien, aunque la explicación de ambas facultades y de su interrelación sigue dependiendo de la psicología empírica de Baumgarten, Kant no entiende el principio de los géneros y las especies meramente como una "regla de la Escuela" o un "principio lógico", ${ }^{11}$ sino más bien como una condición trascendental del conocimiento, pues "no sería posible experiencia alguna" 12 si éste no fuera presupuesto. ${ }^{13}$ $\mathrm{Si}$ en que el uso empírico del entendimiento se sirve de la actividad del ingenio para

\footnotetext{
7 V-Anth/Parow, AA 25, 341.

${ }^{8} \mathrm{KrV}, \mathrm{A} 654 / \mathrm{B} 682$.

9 Ibidem, A 653/ B 681.

${ }_{10}$ Ibidem, A 654/ B 682.

${ }^{11}$ Ibidem, A 652/ B 680.

12 Ibidem, A 654/ B 682.

${ }^{13}$ Sobre el significado del complejo Apéndice a la Dialéctica Trascendental, véase el excelente estudio de Miguel Ángel Santos Gracia. "El "Apéndice a la Dialéctica transcendental" de la Crítica de la razón pura como pars contruens y como teoría de la ciencia". Teorema, 23, 2004, 199-213. El autor sostiene una doble utilidad del concepto de unidad sistemática de la razón defendido por Kant en este texto. En primer lugar, se trataría de aportar la "orientación del entendimiento en la formulación y valoración de hipótesis empíricas"; en segundo lugar, Kant pretendería donar "a las ciencias meramente empíricas (clasificatorias) del único esqueleto racional a priori del que disponen: el de un sistema completo de sus conceptos como ideal regulativo." (207).
} 
descubrir la unidad en un género a partir de la comparación y conexión de fenómenos aislados y aparentemente inconexos, la agudeza posibilita el desarrollo de una investigación empírica de las diferentes especies dentro de cada género. Kant entiende que este proceso de conocimiento se ve guíado por la orientación de la razón especulativa. A este respecto, la actividad del ingenio concuerda con el "interés" racional de descubrir un concepto universal a partir de los datos particulares de la experiencia, lo cual ha de servir al uso empírico del entendimiento.

Este principio (de la agudeza, o facultad de discernimiento) limita mucho la ligereza del primero (del ingenio), y la razón muestra aquí dos intereses que se contradicen entre sí; por un lado, el interés en la extensión (de la universalidad) en relación con los géneros; por otro lado, el interés del contenido (de la determinación) con la intención puesta en la multiplicidad de las especies, porque el entendimiento en el primer caso piensa mucho bajo conceptos, pero en el segundo piensa tanto más en los mismos..$^{14}$

Es cierto que en la Crítica de la razón pura no encontramos un análisis trascendental del ingenio en tanto que una facultad de conocer específica, de tal modo que no es posible arrojar luz sobre su naturaleza y función en el sistema crítico del conocimiento. Pero sí parece claro que Kant le atribuye a este concepto una función en el marco general del uso hipotético de la razón, en la medida en que el significado trascendental del principio de los géneros y las especies tiene lugar en la exposición de esta teoría. Según Kant, en el uso especulativo de la razón lo universal se encuentra ya dado al entendimiento, de tal modo que lo particular puede ser subsumido de forma necesaria a una ley particular ya dada. Ahora bien, si en el proceso de conocimiento sólo el particular se encuentra dado, de tal modo que deba aún hallarse una ley universal a la que subsumirlo, que en un principio sólo puede ser admitida de forma provisional e hipotética, entonces la razón sólo puede orientar el uso empírico del entendimiento de forma hipotética, guiando el proceso de conocimiento mediante ideas regulativas que posibiliten de forma heurística una orientación sistemática y normativa de los conocimientos empíricos. ${ }^{15}$ Se trata entonces del uso hipotético de la razón.

Esta división entre dos aspectos diferentes de la razón nos recuerda a la diferencia general entre la actividad determinante y la actividad reflexionante del Juicio que Kant expondrá nueve años más tarde en la Crítica del Juicio. ${ }^{16}$ Sin embargo, la Dialéctica Trascendental de la primera Crítica no explica aún en qué sentido pueda

\footnotetext{
${ }^{14}$ Ibidem, A 654/ B 683.

15 Ibidem, A 646s./ B 674s.

${ }^{16} \mathrm{KU}$, AA 05, 179: "El Juicio es en general la facultad de pensar lo particular en tanto que contenido bajo lo universal. Si lo universal (la regla, el principio, la ley) se encuentra dado, entonces el Juicio que subsume lo particular a éste [...] es determinante. Pero si sólo lo particular se encuentra dado, para el cual se debe buscar lo universal, entonces el Juicio es meramente reflexionante."
} 
pensarse la orientación normativa de la razón en tanto que un principio específico del Juicio. En la filosofía crítica, considerada desde el nivel de madurez alcanzado en la Crítica del Juicio, este principio sería la ratio essendi del Juicio reflexionante en tanto que facultad de conocer autónoma, y la crítica de los juicios de gusto, en tanto que juicios estético-reflexionantes, sería la ratio cognoscendi de este principio; pues, según Kant, exclusivamente a partir de esta crítica puede demostrarse que el Juicio es una facultad de conocer específica e independiente dentro del sistema crítico de la razón. ${ }^{17}$

A este respecto, el ingenio no es todavía el Juicio reflexionante, aun cuando hemos de admitir que las reflexiones de Kant sobre el ingenio representan uno de los precedentes principales en la historia evolutiva de la formación de la teoría del Juicio reflexionante; un precedente que, además, pone de manifiesto tanto el trasfondo histórico de esta teoría de la tercera Crítica en el contexto de la psicología empírica de Baumgarten como su vinculación sistemática con el concepto de uso hipotético de la razón que es expuesto en la Dialéctica Trascendental. Profundicemos en estas cuestiones.

\section{El ingenio en el Legado póstumo manuscrito y los Apuntes de Lecciones de Antropología}

Los materiales de las Reflexiones del Legado póstumo manuscrito y los Apuntes de Lecciones ponen de manifiesto la relación entre la descripción psicológica del ingenio y la temática expuesta en la Dialéctica Trascendental que ha sido objeto del parágrafo anterior.

\footnotetext{
${ }^{17}$ EEKU, AA 20, 244: "Sólo en el gusto, a saber, en relación con los objetos de la naturaleza, se pone de manifiesto el Juicio en tanto que una facultad que tiene su principio propio, por medio de lo cual reclama con fundamento un lugar en la crítica general de las facultades superiores de conocer del que quizá no se le creía capaz.” Ibidem, AA 20, 225: “[...] de este modo, el juicio estético también reclama que su fundamento de determinación no sólo debe residir en el sentimiento de placer y displacer por sí sólo, sino también en una regla de las facultades superiores de conocer, a saber, en el Juicio, el cual, por lo tanto, es legislador a priori y demuestra autonomía en lo que respecta a la reflexión a priori." La influencia del concepto de "ingenio" en el significado filosófico de la teoría del Juicio reflexionante es sobreestimada cuando se afirma que "der 'Witz' der reflektierenden Urteilskraft ist der Witz" (Gottfried GABRIEL. "Der 'Witz' der reflektierenden Urteislkraft". En R. Rodi (ed.). Urteilskraft und Heurisktik in den Wissenschaften. Beiträge zur Entstehung des Neuen, Velbrück, 2003,197-210: 197). La declaración de Gabriel juega intencionadamente con la equivocidad del concepto en alemán, y podría traducirse como "el quid del Juicio reflexionante es el ingenio." Sin embargo, este quid o clave del Juicio reflexionante sólo puede ser el juicio estético, si atendemos al significado trascendental de la crítica del mismo en el contexto de la deducción de los principios del Juicio reflexionante en general que tiene lugar en la Crítica del Juicio.
} 
El ingenio es una facultad que pertenece a la imaginación productiva o facultad creativa,$^{18}$ y se encuentra al servicio de la invención de nuevas relaciones o el descubrimiento de semejanzas entre las representaciones sensibles. Mientras que la imaginación productiva puede producir nuevas representaciones según su forma, el ingenio produce de forma tentativa nuevas relaciones entre tales representaciones al dar lugar a ocurrencias, establecer analogías o inventar ejemplos. ${ }^{19}$

Ahora bien, esta actividad del ingenio sólo puede ser útil en el proceso de conocimiento si se encuentra bajo el control crítico del Juicio: el ingenio es "una facultad de conocer positiva, de una gran ampliación de nuestros conocimientos. El Juicio es una facultad negativa, una limitación de nuestros conceptos, al mostrar que un concepto no se aplica a tantas cosas como se piensa." ${ }^{20} \mathrm{El}$ ingenio fomenta un juego libre ${ }^{21}$ de las representaciones, que por tal motivo pueden combinarse de forma voluntaria y provisional. De este modo, a través del trabajo conjunto y de la complementación de ambas facultades, el entendimiento recibe un material rico para el conocimiento, en la medida que facilita el hallazgo de nuevos conceptos y nuevas reglas en la investigación de la naturaleza. ${ }^{22}$ Las ocurrencias o invenciones del ingenio pueden inducir a error, pues éste no atiende a las diferencias de las representaciones entre sí ni limita o corrige aún sus imágenes según intelecciones o conocimientos ya determinados. Tal intelección determinante es aportada por el Juicio, que debe poner a prueba la corrección de las ocurrencias del ingenio, así como detectar en detalle las diferencias en el interior de los conceptos que se han bosquejado provisionalmente por el ingenio. Mientras que esta última facultad proporciona al entendimiento una regla general caracterizada por la laxitud, el Juicio debe "subsumir si algo pertenece a un concepto universal o no"23. Por lo tanto, el Juicio es aquí entendido en tanto que facultad determinante, que limita la equivocidad de las imágenes del ingenio con el objetivo de poner a prueba la utilidad que pueda tener para el conocimiento el material aportado provisionalmente por el

\footnotetext{
${ }_{18}$ Cf. V-Anth/Pillau, AA 25, 754; Refl. 329, aprox. 1776-1778 (?), AA 15, 130. En la V-Menschenkunde Kant define la imaginación productiva como facultad creativa, que se corresponde con el fundamento de toda invención de nuevos conocimientos [AA 25, 981]. Véase también V-Anth/Friedländer, AA $25,524$.

${ }^{19}$ Cf. V-Anth/Fried, AA 25, 516s.; V-Menschenkunde, AA 25, 959; V-Anth/Mron, AA 25, 1263.

${ }_{20} V$-Menschenkunde, AA 25, 959. Véase también V-Anth/Mron, AA 1263; V-Anth/Busolt, AA 25, 1459.

${ }^{21} V$-Anth/Busolt, AA 25, 1459: "El ingenio [Wiez] divierte a través del juego libre, a diferencia del Juicio, que sin embargo es agradable. El ingenio es libre; el Juicio limita." La caracterización de la actividad del ingenio como juego también la encontramos en V-Anth/Pillau, AA 25, 754; V-Menschenkunde, AA 25, 969, 1063; V-Anth/Mron, AA 25, 1266-68, y procede históricamente de la misma exposición de Baumgarten, Metaphysica..., § 576, AA 15, 23.

${ }_{22}$ Cf. Anth/Fried, AA 25, 517; V-Anth/Pillau, AA 25, 754s.; V-Menschenkunde, AA 25, 959; V-Anth/ Mron, AA 25, 1263-65; V-Anth/Busolt, AA 25, 1459s.

${ }^{23}$ Cf. V-Anth/Pillau, AA 25, 754.
} 
ingenio: "Mediante el juzgar nos guardamos de los errores. El Juicio se dirige a la rectificación (corregir, explicar)"24. Por lo tanto, se trata de un "tipo de disciplina para el ingenio" 25 , por la que es posible comprobar si las imágenes y los conceptos bosquejados por este último pueden aplicarse a los objetos in concreto, una vez que el Juicio discierne su diferencia específica, de tal modo que puedan convertirse en conceptos determinados del entendimiento.

Aunque las exposiciones sobre el ingenio y el Juicio en los Apuntes de Lecciones de Antropología tienen sin duda un carácter psicológico, el trabajo conjunto y complementario de ambas facultades constituye el procedimiento cognoscitivo subjetivo que posibilita la investigación empírica de los géneros y las especies en el conocimiento.

El ingenio intenta formar un concepto a partir de las diferentes representaciones comparadas, y a través del Juicio diferenciamos las especies que se encuentran contenidas bajo el género. Nuestro conocimiento crece más mediante el ingenio, al poder hacerlo universal por medio de éste. Tal concepto es de gran alcance, y puedo aplicarlo a muchos objetos. Hacemos géneros a partir de él, que contienen las semejanzas de muchas cosas. ${ }^{26}$

La función conjunta que desempeñan ingenio y Juicio en la formación del conocimiento empírico según géneros y especies refleja en el plano de los Apuntes la descripción de la función que desempeñaban ingenio y agudeza en el Apéndice de la Dialéctica Trascendental, donde Kant expone el principio lógico de los géneros y las especies en tanto que una idea trascendental, que debe ser presupuesta en la investigación empírica a partir del interés especulativo de la razón teórica. Ciertamente, esta dimensión trascendental implicada en el proceso cognoscitivo no es tematizada explícitamente en los Apuntes, pero Kant sí defiende que la invención, actividad propia del ingenio, debe encontrarse en relación con las ideas de la razón.

Esta facultad creativa es la base de todas las invenciones; siempre probamos a producir nuevas representaciones; a continuación, el entendimiento debe probarlas para reorganizarlas y que se encuentren en relación con las ideas de la razón. ${ }^{27}$

Ahora bien, la facultad creativa [Dichtungsvermögen], en tanto que facultad de la invención y la creación en general, es el fundamento del ingenio. Y la invención [Erfinden] es además el campo en el que desempeñan su función los juicios provisionales [vorläufige Urteile], cuyo significado regulativo y heurístico los sitúa en

${ }^{24}$ Cf. V-Menschenkunde, AA 25, 959.

25 V-Anth/Pillau, AA 25, 755.

${ }_{26} V$-Menschenkunde, AA 25, 959s. Véase también $V$-Anth/Busolt, AA 25, 1459; V-Anth/Mron, AA 25, 1266s.; Refl. 469, 1776-1778, AA 15, 194.

27 V-Menschenkunde, AA 25, 981. 
el ámbito del uso hipotético de la razón. Un juicio provisional es un juicio problemático que precede a la investigación, con el objeto de indicar la vía o el camino a seguir a partir de máximas de tipo heurístico. ${ }^{28}$ Pues, tal como ya demuestra la misma Dialéctica Trascendental, el proceso de conocimiento no sólo es posible bajo el presupuesto de las categorías del entendimiento: "Por este motivo, se bosqueja una red de suposiciones, que ha de posibilitar que la reflexión no se convierta en un mero 'andar a tientas' [Herumtappen]. ${ }^{\prime 29}$ En conformidad con la exposición de Kant en las Lecciones de Enciclopedia, hay juicios provisionales que se basan en las mismas ideas de la razón, pues no son propiamente juicios, sino más bien máximas heurísticas para el enjuiciamiento, es decir, principios regulativos de la reflexión para el enjuiciamiento, a través de los cuales no se determina objeto alguno..$^{30}$ Entre las máximas de la reflexión para la investigación de la naturelaza debemos contar la de no interpretar los juicios provisionales como si fuesen juicios determinados. Los juicios provisionales son más bien propuestas previas que no se encuentran aún determinadas, que preceden a los juicios determinados. ${ }^{31}$ Esta misma exigencia es también condición para el uso del ingenio en el proceso de conocimiento, pues, en el ámbito del conocimiento, los conceptos e imágenes propuestos por esta facultad sólo pueden ser aceptados de forma provisional, al menos hasta que el Juicio haya determinado su corrección y su diferencia específica. Es más, los juicios provisionales son producidos por el ingenio. Por lo tanto, el sujeto debe reflexionar sobre los juicios provisionales del ingenio con el objeto de conciliarlos con la determinación del Juicio y, de este modo, autorizarlos como juicios determinados:

\footnotetext{
${ }^{28}$ Cf. K. ReISInger. "Urteil, vorläufig”. En Joachim Ritter et alii (ed.). Historisches Wörterbuch der Philosophie. Darmstadt, 1971ss. (2001), XI 473-79: 473. El concepto lo desarrolla Kant en el contexto del debate ilustrado sobre los prejuicios; sobre el transfondo histórico de esta teoría, véase Norbert HinsKe. "G. F. Meier und das Grundvorurteil der Erfahrungserkenntnis". En Claudio Cesa y Norbert Hinske (ed.). Kant und sein Jahrhundert. Frankfurt am Main, 1993, 103-21.

${ }^{29}$ Claudio LA RoccA. "Vorläufige Urteile und Urteilskraft. Zur heuristischen Logik des Erkenntnisprozesses". En Volker Gerhardt et alii (ed.). Kant und die Berliner Aufklärung. Akten des IX. Internationalen Kant-Kongresses. Berlin y New York, 2001, II 351-61. En otro trabajo, el autor ha señalado que el tratamiento del concepto de juicio provisional se enmarca también en el contexto general del conocimiento inconsciente y las representaciones oscuras; véase Claudio LA RoccA. "L'intelletto oscuro. Inconscio e autocosciencia in Kant". En Claudio La Rocca (ed.). Leggere Kant. Dimensioni della filosofia critica. Pisa, 2007, 63-116: 84s.

${ }^{30}$ La Rocca, "Vorläufige Urteile", 354. Por este motivo, La Rocca ha defendido que los juicios provisionales son un precedente importante del concepto de Juicio reflexionante, especialmente en lo que respecta a la comprensión de la validez intersubjetiva de la reflexión; veáse también LA RoccA, "L'intelletto oscuro", 85.

${ }^{31}$ La RoccA, "Vorläufige Urteile", 356: "Andererseits stellt sich hier die Frage, wie die vorläufigen Urteile reflexiv geregelt werden. Das geschieht [...] zunächst durch das Bewusstsein ihrer provisorischen Natur, das ihre Verwandlung in bestimmende Urteile verhüten soll."
} 
[Un] juicio provisional es un fundamento para juzgar sobre cosas, el cual sin embargo es insuficiente. Pero emitir un juicio determinado es algo propio del Juicio. El ingenio merodea sin orden allí donde encuentra algo; y por lo tanto también sirve para la invención, pero por este motivo también induce a errores, lo cual ocurre, pues, cuando no se quiere reflexionar sobre el ingenio y sus juicios provisionales para conciliarlos con el Juicio. Pero el Juicio sirve al juicio determinado, y por este motivo lo mantiene libre de errores. ${ }^{32}$

Este texto no sólo es importante por ofrecernos una mejor comprensión del concepto de ingenio, sino especialmente porque en él se explícita la vinculación que existe entre este concepto y la teoría de los juicios provisionales, que no es manifiesta en los textos de la obra crítica, a pesar de que ambos aspectos de la reflexión de Kant se sitúan en el marco general de la teoría del uso hipotético de la razón y deben ser considerados como momentos fundamentales del desarrollo histórico-evolutivo de la teoría del Juicio reflexionante. Por un lado, Kant explica el ingenio como la facultad de bosquejar o formar diferentes géneros a partir de la comparación de las representaciones inicialmente inconexas, a través de lo cual el proceso empírico de conocimiento puede desarrollarse según una idea sistemática de la naturaleza que es aportada regulativamente por la razón teórica. Por otro lado, esta función del conocimiento presupone las máximas de la reflexión que prescriben al sujeto considerar los propios conocimientos como provisionales, al menos hasta que el Juicio (determinante) haya inteligido, corregido y, en definitiva, determinado tales conocimientos.

Pues bien, el ingenio no sólo se encuentra en relación con la teoría del uso hipotético de la razón, sino con la futura teoría del Juicio reflexionante. Esta vinculación temática entre el ingenio y el Juicio reflexionante es manifiesta en la definición del primero que Kant aporta en la Antropología, planteada mediante un paralelismo con respecto al Juicio reflexionante.

Del mismo modo que el Juicio es la facultad de encontrar lo particular para lo universal (de la regla), el ingenio (ingenium) es la [facultad] de encontrar lo universal para el particular (ingenium). ${ }^{33}$

Si se atiende a sus definiciones generales, 'Juicio reflexionante' y 'Juicio determinante' se corresponden en la tercera Crítica con los conceptos 'ingenio' y 'Juicio' en la Antropología en sentido pragmático. No obstante, en la medida en que el texto citado data de 1798, la declaración contenida en este texto no nos autoriza aún a admitir que el concepto de ingenio haya jugado algún papel en el surgimiento de la teoría del Juicio reflexionante con anterioridad a la publicación de la Crítica del Juicio.

32 V-Anth/Friedländer, AA 25, 516; véase también V-Menschenkunde, AA 25, 968.

${ }^{33}$ Anth., AA 07, 201. 


\section{Ingenio y Juicio reflexionante}

El problema del Juicio reflexionante se expone en la tercera Crítica como la cuestión de cómo sea posible hallar un concepto universal del entendimiento para un particular dado a la sensibilidad, a través del cual determinar éste en un sistema de conocimientos. En sus reflexiones con respecto al proceso de enjuiciamiento por el que se busca establecer esta relación entre lo particular y lo universal, la atención de Kant se centra en la posibilidad de una conformidad subjetiva entre facultades de conocer implicadas en el enjuiciamiento y el problema de su heterogeneidad. El enjuiciamiento supone una conciliación entre sensibilidad o imaginación, de un lado, y entendimiento en general, del otro.

Si bien debemos esperar a la publicación de la Crítica del Juicio para encontrar la formulación de una teoría fundada sobre la relación entre las facultades en el enjuiciamiento reflexionante, Kant habría atendido a este problema con anterioridad a 1790, precisamente debido a su interés por desarrollar una solución sobre las condiciones que rigen esta capacidad. Pues bien, debe constatarse que en el contexto de estas reflexiones, que conducirán al progresivo desarrollo de la teoría del Juicio reflexionante, Kant caracteriza el ingenio como la capacidad del ánimo que funciona en tanto que vínculo entre la imaginación y el entendimiento, en la medida en que posibilita la formación provisional de un universal desde la misma sensibilidad, y cuyos productos pueden ser determinados definitivamente por el entendimiento para el conocimiento mediante la labor crítica del Juicio (determinante). ${ }^{34}$

La imaginación contiene en sí la compilación del conocimiento sobre todo aquello que hemos percibido, pensado o tratado. El fin al cual se dirige todo esto es el entendimiento. Se trata de dos facultades para emplear los materiales que residen en la imaginación y aplicarlos al servicio del entendimiento: ingenio y Juicio. ${ }^{35}$

Ingenio y Juicio son facultades para aplicar la imaginación al servicio del entendimiento. El primero, para bosquejar todo lo que encaja en ello; el segundo, para llevar a cabo entre la multiplicidad una elección de lo que sea conforme al concepto del entendiminento. Lo que corresponde. ${ }^{36}$

La descripción psicológica de la relación entre imaginación y entendimiento que haya de dar lugar a una conformidad o coincidencia para la formación de un conocimiento en general se apoya en los conceptos de ingenio y Juicio (determinante), a los que Kant ha dirigido su atención desde principios de la década de 1770 . Se

\footnotetext{
${ }^{34}$ En su análisis de las Lecciones de Antropología, SATURA, Kants Erkenntnispsychologie, 65-82, no tematiza el papel del ingenio y del Juicio en relación con el problema de la relación de las facultades y la formación de los conceptos empíricos.

35 V-Anth/Pillau, AA 25, 754.

${ }^{36}$ Refl, 477, 1776-1779, AA 15, 197. Véase también V-Anth/Mron, AA 25, 1268.
} 
trata del problema de la formación de conceptos, definido por aquella situación en que la facultad de conocer debe encontrar una guía para determinar un particular, la cual no puede ser aportada por un concepto previo, pues la necesidad de este proceso adquiere sentido precisamente por la ausencia de tal concepto determinante y la exigencia de hallarlo. Ahora bien, nos encontramos en el terreno de la descripción psicológica o antropológica de la relación entre las facultades.

En la tercera Crítica, por el contrario, Kant defenderá la tesis de que en esta interrelación entre las facultades de conocer que se exige para el enjuiciamiento de la naturelaza debe presuponerse a priori un principio trascendental y subjetivo, que el Juicio reflexionante se da a sí mismo para la reflexión sobre los particulares y su posible determinación en el sistema del conocimiento. Ahora bien, esta fundamentación del Juicio reflexionante en tanto que una facultad de conocer especifica en el sistema de la razón sólo es expuesta en la obra por mediación de un análisis crítico y una deducción de aquellos juicios en que se manifiesta de forma ejemplar el problema de la reflexión subjetiva: los juicios estéticos.

Desde los fundamentos y límites establecidos en la primera Crítica, la determinación crítica de la diferencia específica entre sensibilidad y entendimiento implica por principio la imposibilidad de suponer o demostrar a priori la conformidad entre ambas facultades. De este modo, el principio del Juicio reflexionante no debe interpretarse como un precepto desde el que asegurar a priori, por ejemplo, que el contenido de la sensibilidad podrá encontrar su integración en un sistema de conocimientos según el orden definido previamente por el espacio lógico que determinan los conceptos empíricos del entendimiento. Esta correspondencia objetiva, que define la verdad material de los juicios, sólo puede comprobarse a posteriori. Desde el punto de vista de las facultades esto significa que el principio del Juicio no puede determinar a priori la conformidad entre sensibilidad y entendimiento en lo que respecta a la verdad material de los juicios; el principio del Juicio sólo puede legislar a priori en la medida en que se reconoce que esta legislación no tiene validez objetiva. Esta característica del enjuiciamiento reflexionante en general es manifiesta especialmente en los juicios estéticos de gusto, en los que una regla estética nunca puede servir para mostrar a priori qué representación provocará un sentimiento de placer en nuestro ánimo y, por tanto, será considerada como bella. Ahora bien, en el caso de que el sujeto sienta (a posteriori) un placer universalmente comunicable con ocasión del tratamiento reflexionante de una representación, entonces la crítica debe admitir a priori que el fundamento de este sentimiento ha de residir en aquello que hay de univesal en el Juicio reflexionante, a saber, en un principio subjetivo que guía la actividad de las facultades en la reflexión sobre los objetos, que es definido por la crítica como una condición necesaria del conocimiento en general. ${ }^{37}$

37 Véase por ejemplo EEKU, 20, 230-32; KU, AA 05, 219-22, 286-91. 
No es posible profundizar aquí en la compleja argumentación con la que Kant desarrolla la deducción trascendental de los juicios de gusto en la tercera Crítica, como tampoco en los diferentes estadios de génesis y formación de esta argumentación con anterioridad a esta obra. Baste con señalar aquí que un bosquejo de esta argumentación sobre la aprioridad del juicio de gusto puede localizarse ya en la Refl. 988, que data aproximadamente de $1784 .{ }^{38}$ Aunque en este texto Kant no emplea aún el concepto de Juicio 'reflexionante', sí expone la concepción de que el análisis del sentimiento de placer en el gusto pone de manifiesto la condición subjetiva del conocimiento en general, que es presupuesta por las facultades de conocer implicadas en el enjuiciamiento. En la Crítica del Juicio Kant entiende por tales facultades solamente la imaginación y el entendimiento, sin explicitar la intervención del ingenio como componente de la creatividad o productividad de la imaginación, y a diferencia de los Apuntes de Lecciones, en los que sí es manifiesto el papel desempeñado por el ingenio en el proceso de conocimiento. Ahora bien, en la Refl. 988 Kant cuenta el ingenio entre las facultades de conocer cuya actividad presupone un fundamento subjetivo del conocimiento en el enjuiciamiento de la representación.

Las facultades de conocer son ingenio e imaginación, en la medida que son conformes con el entendimiento. El Juicio sólo es la facultad que posibilita la coincidencia de éstos ( ${ }^{\mathrm{g}}$ en un caso) in concreto. Agudeza es la facultad por la que también se detecta la menor coincidencia o conflicto entre éstos; es, por lo tanto, una propiedad del Juicio. ${ }^{39}$

Aunque la Refl. 988 bosqueja la estructura argumentativa que se empleará en la deducción de los juicios estéticos de gusto y, con ello, de los principios presupuestos en general en el Juicio reflexionante, Kant emplea aún en este texto la terminología procedente de la psicologia empírica de Baumgarten, con la que había enlazado en los inicios de su desarrollo intelectual y a través de la cual reflexionaba sobre la formación sistemática del conocimiento empírico en el marco de su actividad académica desde 1772.

El ingenio no es el Juicio reflexionante, que sólo en la Crítica del Juicio será considerado por Kant como una facultad autónoma en el sistema de la razón pura, al fundamentar la validez subjetiva de sus principios mediante el análisis crítico del gusto. Sin embargo, el conocimiento de la historia evolutiva del concepto 'Witz' nos

${ }_{38}$ Refl. 988, 1783-1784, AA 15, 432s. Este texto debe considerarse como el primer bosquejo de la futura deducción del gusto. Un desarrollo de lo que aquí se plantea lo encontramos en la Refl. 992, 17861789. Véase al respecto, Piero GiordanetTI. "Kants Entdeckung der Apriorität des Geschmacksurteils. Zur Genese der Kritik der Urteilskraft". En D. Falcioni et alii (ed.). Aufklärung und Interpretation. Tagung aus Anlaß des 60. Geburtstag von Reinhard Brandt. Würzburg, 1999, 171-96; así como M. SÁnchez Rodríguez. Sentimiento y reflexión en la filosofía de Kant. Estudio histórico del problema estético. Hildesheim, Zürich y New York: 192-209.

${ }^{39}$ Refl. 988, AA 15, 432. 
acerca a los múltiples aspectos que rodean la problemática que tratará de afrontar la teoría del Juicio reflexionante, especialmente a partir de textos aún poco conocidos por los intérpretes de Kant. En primer lugar, puede observarse que las reflexiones de este pensador en torno al problema del enjuiciamiento a principios de la década de 1770 enlazan con la terminología de la teoría de las facultades que él encontraba en la psicología empírica de Baumgarten, sección que empleaba como manual para impartir sus Lecciones de Antropología. Este enlace no significa que Kant adoptara sin más la concepción de Baumgarten. Más bien, ya desde 1772 había llevado a cabo una explícita corrección de esta teoría. Aunque en la Crítica de la razón pura encontramos aún una postura cercana a la formulación baumgartiana, lo cierto es que en esta obra se afirma la dependencia del ingenio con respecto a las ideas trascendentales de la razón, una perspectiva ausente en la Metafisica de Baumgarten. En segundo lugar, la historia del concepto de ingenio pone de manifiesto la vinculación temática entre la problemática expuesta en el Apéndice a la Dialéctica Trascendental y la teoría del Juicio reflexionante. El Juicio reflexionante tiene aplicación y sentido en el ámbito propio del uso hipotético de la razón, no sólo por aludir al proceso de conocimiento que va de abajo arriba, por así decirlo, sino también por ser un proceso basado en principios puros y subjetivos que residen en última instancia en la razón, a partir de los cuales puede pensarse la naturaleza como una totalidad susceptible de ser conocida progresivamente por el entendimiento. Aunque es común aceptar esta vinculación entre ambas obras, lo cierto es que no existe ninguna declaración de Kant en que se admita que la teoría del Juicio reflexionante trataba de responder a la misma problemática descrita en el Apéndice a la Dialéctica Trascendental. Ahora bien, el concepto de ingenio representa una idea que recorre el desarrollo intelectual de Kant desde 1781 a 1790, y que se encuentra en conexión temática tanto con el Apéndice como con la teoría del Juicio reflexionante. De este modo, este artículo muestra que la vinculación temática a este respecto entre la Crítica de la razón pura y la Crítica del Juicio responde a un interés sistemático de Kant que puede ser rastreado históricamente al menos desde 1781.

Ni que decir tiene que la historia del concepto de ingenio no agota completamente la historia evolutiva del problema estético en el pensamiento de Kant. Incluso en lo que respecta al concepto de Juicio reflexionante, es preciso admitir que en él confluyen diversos problemas, influencias e historias conceptuales. ${ }^{40}$ En este trabajo se ha querido atender a uno de esos momentos que son relevantes para entender la compleja historia evolutiva del proyecto estético de Kant.

\footnotetext{
40 En otro trabajo he defendido la importancia de los conceptos "Mutterwitz" y "gesunder Verstand", procedentes de la logica y la estética de la escuela leibniziano-wolffiana, para comprender la génesis de la teoría del Juicio reflexionante en Kant: Manuel Sánchez Rodríguez. "Logica naturalis, Healthy Understanding and the Reflecting Power of Judgment in Kant's Philosophy. The Source of the Problem of Judgment in the Leibniz-Wolffian Logic and Aesthetics". Kant-Studien, 103, 2012, 188-206.
} 


\section{Bibliografía}

BAeumler, Alfred. Das Irrationalitätsproblem in der Ästhetik und Logik des 18. Jahrhunderts bis zur Kritik der Urteilskraft. Vol. 1: Kants Kritik der Urteilskraft. Ihre Geschichte und Systematik. Tübingen, 1923.

Baumgarten, Alexander Gottlieb. Metaphysica. Halle, 1739 (reimpresión según la cuarta edición de 1757, en AA 15, 5-54 y AA 17, 5-226).

GABRIEL, Gottfried. 'Der 'Witz' der reflektierenden Urteislkraft”. En R. Rodi (ed.). Urteilskraft und Heurisktik in den Wissenschaften. Beiträge zur Entstehung des Neuen, Velbrück, 2003, 197-210.

Giordanetti, Piero. "Kants Entdeckung der Apriorität des Geschmacksurteils. Zur Genese der Kritik der Urteilskraft”. En D. Falcioni et alii (ed.). Aufklärung und Interpretation. Tagung aus Anlaß des 60. Geburtstag von Reinhard Brandt. Würzburg, 1999, 171-96.

Hinske, Norbert. "Kant und Alexander Gottlieb Baumgarten. Ein leider unerledigtes Thema der Anthropologie Kants". Aufklärung, 14, 2002, 261-74.

KAnt, Immanuel. Kant's gesammelte Schriften. Ed. de la Königlich Preußischen Akademie der Wissenschaften und ihren Nachfolgern. Berlin y Leipzig, 1900ss. (= AA).

LA RoccA, Claudio. "Vorläufige Urteile und Urteilskraft. Zur heuristischen Logik des Erkenntnisprozesses". En Volker Gerhardt et alii (ed.). Kant und die Berliner Aufklärung. Akten des IX. Internationalen Kant-Kongresses. Berlin y New York, 2001, II 351-61.

LA RoccA, Claudio. "L'intelletto oscuro. Inconscio e autocosciencia in Kant". En Claudio La Rocca (ed.). Leggere Kant. Dimensioni della filosofia critica. Pisa, 2007, 63-116.

Locke, John. An Essay Concerning Human Understanding. Works of John Locke, 10 vols. London, 1823 ( $\left.{ }^{1} 1690\right)$ (reimpresión en Aalen, 1963).

REISINGER, K. "Urteil, vorläufig”. En Joachim Ritter et alii (ed.). Historisches Wörterbuch der Philosophie. Darmstadt, 1971ss. (2001), XI 473-79.

SÁnchez Rodríguez, Manuel. Sentimiento y reflexión en la filosofía de Kant. Estudio histórico del problema estético. Hildesheim, Zürich y New York, 2010.

SÁnchez Rodríguez, Manuel. "Logica naturalis, Healthy Understanding and the Reflecting Power of Judgment in Kant's Philosophy. The Source of the Problem of Judgment in the Leibniz-Wolffian Logic and Aesthetics". KantStudien, 103, 2012, 188-206.

Santos Gracia, Miguel Ángel. "El “Apéndice a la Dialéctica trasncendental” de la Crítica de la razón pura como pars contruens y como teoría de la ciencia". Teorema, 23, 2004, 199-213.

SATURA, Vladimir. Kants Erkenntnispsychologie in den Nachschriften seiner Vorlesungen über empirische Psychologie. Bonn, 1971. 
Sulzer, Johann Georg. Entwicklung des Gebriffs von Genie. Vermischte philosophische Schriften. Hildesheim, 1974 ('1773), 307-22.

\section{Manuel Sánchez Rodríguez}

Departamento de Filosofía II - Universidad de Granada msr@ugr.es 